\title{
Microwave-assisted fibrous decoration of mPE surface utilizing Aloe vera extract for tissue engineering applications
}

This article was published in the following Dove Press journal:

International Journal of Nanomedicine

18 September 2015

Number of times this article has been viewed

\author{
Arunpandian Balaji \\ Saravana Kumar Jaganathan \\ Eko Supriyanto \\ Ida Idayu Muhamad \\ Ahmad Zahran Md \\ Khudzari \\ Institut Jantung Negara-Universiti \\ Teknologi Malaysia, Cardiovascular \\ Engineering Centre, Faculty of \\ Biosciences and Medical Engineering, \\ Universiti Teknologi Malaysia, Johor \\ Bahru, Malaysia
}

\begin{abstract}
Developing multifaceted, biocompatible, artificial implants for tissue engineering is a growing field of research. In recent times, several works have been reported about the utilization of biomolecules in combination with synthetic materials to achieve this process. Accordingly, in this study, the ability of an extract obtained from Aloe vera, a commonly used medicinal plant in influencing the biocompatibility of artificial material, is scrutinized using metallocene polyethylene (mPE). The process of coating dense fibrous Aloe vera extract on the surface of mPE was carried out using microwaves. Then, several physicochemical and blood compatibility characterization experiments were performed to disclose the effects of corresponding surface modification. The Fourier transform infrared spectrum showed characteristic vibrations of several active constituents available in Aloe vera and exhibited peak shifts at far infrared regions due to aloe-based mineral deposition. Meanwhile, the contact angle analysis demonstrated a drastic increase in wettability of coated samples, which confirmed the presence of active components on glazed mPE surface. Moreover, the bio-mimic structure of Aloe vera fibers and the influence of microwaves in enhancing the coating characteristics were also meticulously displayed through scanning electron microscopy micrographs and Hirox 3D images. The existence of nanoscale roughness was interpreted through high-resolution profiles obtained from atomic force microscopy. And the extent of variations in irregularities was delineated by measuring average roughness. Aloe vera-induced enrichment in the hemocompatible properties of mPE was established by carrying out in vitro tests such as activated partial thromboplastin time, prothrombin time, platelet adhesion, and hemolysis assay. In conclusion, the Aloe vera-glazed $\mathrm{mPE}$ substrate was inferred to attain desirable properties required for multifaceted biomedical implants.
\end{abstract}

Keywords: Metallocene polyethylene, fibrous coating, physicochemical properties, blood compatibility

\section{Introduction}

Aloe vera is one of the familiar plants that has been unceasingly used in the day-to-day life of human beings. Its nutrients and active constituents have helped humankind to overcome a spectrum of ailments ranging from wound healing to malignancy for several centuries. Basically, it is a short-stemmed, perennial, drought-resisting, succulent plant that contains hundreds of potentially active substances in the form of essential amino acids, glucomannans, minerals, lipids, vitamins, polysaccharides, major polypeptides, proteins, and antioxidants. ${ }^{1,2}$ These substances have gained Aloe vera a favorable reputation in cosmetic, health care, and food industries. At present, the total market value of Aloe vera and its nutrients is about 35 billion US dollars, and it is being cultivated in several countries for commercial purposes. ${ }^{3}$ On the other hand, 
extensive research performed to scrutinize the therapeutic aptitude of Aloe vera has revealed its habit of encouraging tissue regeneration or rehabilitation by improving oxygen and blood supply, shielding microbial attacks, and providing essential nourishments..$^{47}$ Recent works reported on the use of Aloe vera in the synthesis of modern materials such as nanoparticles, nanocomposites, and nanofibers have proved its importance in the field of wound healing, tissue regeneration, and cancer drug delivery. ${ }^{8-13}$ Inspired by its profound background, in this work, the extract obtained from Aloe vera is utilized to develop an antifouling and biocompatible material for multipurpose biomedical implants for tissue engineering applications.

Bio-implants are man-made systems intended to assist or replace the functions of biological parts. Nowadays, the need for durable bio-implants has been mounting steadily in response to the increase in incidence of age-related chronic illnesses like osteoarthritis, cardiovascular diseases, and congenital and neurological disorders. Because of this, the global market of bio-implants ranging from cardiovascular to urological disorders is expected to rise from 94.1 billion US dollars in 2012 to over 134.3 billion US dollars by 2017 , assuming compound annual growth rate of $7.3 \%$. Further, the fact of an increasing ageing population around the world is anticipated to highly influence the statistics mentioned earlier. According to Centers for Disease Control and Prevention, the United States accommodated more than 40 million people in the age group of 65 years and older in 2010. If it continues in this manner, the total population of United States will comprise $>20 \%$ of aged people by $2030 .{ }^{14,15}$ Apart from this, the poor lifestyle habits are creating a platform for young people to become prey for several age-related ailments. So, sooner or later, the above-mentioned causes will create huge demand for reliable bio-implants, especially in the cardiovascular, dental, and orthopedic fields.

Basically, the implants are made of metals, ceramics, polymers, and composites, which are collectively called biomaterials. Polymers have evolved as the most fascinating choice because of their commendable mechanical properties and easy-to-tailor nature. At present, polymers have a spectrum of applications in the medical arena, ranging from artificial prostheses to drug delivery systems. Though polymers have reasonable advances when compared with their fellow materials, they fail on several occasions due to the problem of blood compatibility. ${ }^{16,17}$ In addition, rejection of biomaterials is also reported to occur due to cytotoxicity and microbial attacks. So, an ideal material is one that can combat the expected complications, and this property is termed as biocompatibility. In fact, to improve the biocompatibility of a material, several modification techniques have been formulated and demonstrated. ${ }^{18,19}$

In a different trend, the ability of Aloe vera in influencing the biocompatibility of a material is demonstrated using metallocene polyethylene (mPE). It is one of the polyolefin polymers synthesized using a metallocene catalyst, and $\mathrm{mPE}$ has been utilized for several basic applications in clinical industry. It is reported to possess commendable mechanical properties; further, it is cost-effective and displays excellent film density, which makes it a suitable replacement choice for several expensive medical materials in practice. ${ }^{20-22}$ However, the problem of biocompatibility associated with $\mathrm{mPE}$ is hampering its exploitation in the field of biomedical implants. Accordingly, recent efforts made to solve this problem by manipulating surface modification techniques like radiation (microwave) and acid etching have yielded encouraging results and also expressed its capability to act as a promising biomaterial. ${ }^{23,24}$ In this study, an effort has been made to improve biocompatibility and to impart the integrity of Aloe vera on the mPE surface to make it suitable for tissue engineering applications.

The aptitude of artificial materials to promote and support the tissue rehabilitation mechanism is highly desired. So, the surface tailoring techniques are always anticipated to create a wholesome platform to satisfy all the aspects of biocompatibility. This can be achieved by exploiting common biological substances like proteins, lipids, peptides, polysaccharides, antioxidants, etc. Scientists have already demonstrated the role of these biomolecules in enhancing the quality of several polymeric materials by delaying clot formation, reducing platelet adhesion followed by activation, and shielding microbial attacks. ${ }^{25-29}$ On the other hand, Aloe vera extract utilized in this work is a natural storehouse of several vital substances required to promote and guide the regeneration mechanism. Therefore, the competence of Aloe vera extract in repairing the surface characteristics and enhancing the blood compatibility of mPE was scrutinized.

\section{Experimental section Materials}

Chemicals required for performing activated partial thromboplastin time (APTT), prothrombin time (PT), hemolysis, and platelet adhesion tests were purchased from Sigma-Aldrich (St Louis, MO, USA) and utilized without further purification. Sheets of mPE were kindly offered by Indian Institute of Technology, Kharagpur, India. Blood samples and freshly prepared platelet-rich plasma (PRP) required for performing blood compatibility assays were obtained from Dindigul Blood Bank, Dindigul, India. Blood was taken from healthy 
adult human volunteers according to the predetermined protocol of Dindigul Blood Bank. Further, all the procedures involved in the usage of blood were approved and overviewed by the Institutional Ethical Committee at PSNA College of Engineering and Technology, Dindigul, India.

\section{Isolation and preparation of Aloe vera extract}

Initially, fresh succulent leaves of Aloe vera were carefully handpicked and washed with distilled water. Then, the skinny upper part and the yellowish latex part were peeled and discarded. This was followed by the separation of semitransparent whitish gel with the help of a metal ladle. Finally, the gel was blended into a concentrated fibrous extract, which was used for coating mPE samples without further alteration.

\section{Microwave-assisted Aloe vera coating}

Metallocene polymer sheet was cut into identical squareshaped samples of dimension $2 \mathrm{~cm} \times 2 \mathrm{~cm}$ and washed with distilled water and $70 \%$ ethanol to get rid of foreign particles (if any) on the surface. Before coating, the samples were treated with microwaves; the ability of microwaves in accelerating the chemical process and their role in the development of homogeneous coating have been documented already. ${ }^{30-33}$ However, in this work, we followed a unique protocol to facilitate effective coating of Aloe vera on mPE samples by energizing the polymer surface and improving its roughness with the help of microwaves. ${ }^{23}$

Initially, samples to be coated were exposed to microwave of $800 \mathrm{~W}$ and 2,450 MHz produced from Samsung ME711K microwave oven for a period of 60 seconds.
Then the samples were submerged in sterilized petri dishes filled with 10-15 mL of prepared Aloe vera extract. To smoothen the coating process, Aloe vera-filled vessels were placed on the rocking shaker providing seesaw motion at constant speed and maintained under optimum conditions. The samples were removed at two different times, that is, one set of samples was taken out from the petri dish after 12 hours, while the other set was retrieved after 24 hours. Both Aloe vera-glazed samples were dried at room temperature overnight to eliminate the moisture present on the surface. Further, for all surface and blood compatibility characterization studies, samples were freshly prepared according to the described protocol. The results of 12 hours Aloe vera-coated (A-12 h-mPE) and 24 hours Aloe vera-coated (A-24 h-mPE) samples were compared with pristine-control $\mathrm{mPE}$. The process of extracting, preparing, and coating Aloe vera extract on $\mathrm{mPE}$ substrates is presented in Figure 1.

\section{Surface characterization studies}

Fourier transform infrared (FTIR) spectroscopic analysis was performed for both control and coated samples using IRTracer-100 (Shimadzu) system, and the spectrum was recorded over the range of $400-4,000 \mathrm{~cm}^{-1}$ at 40 scans per minute. The changes in wettability of mPE surfaces after coating with Aloe vera were demonstrated using VCA Optima contact angle measurement unit. A water droplet of size $2 \mu \mathrm{L}$ was used; since the surfaces were highly hydrophilic, the photographs were taken immediately after placing the droplet (within 10 seconds) using ultrafast mode. Further, the degree of angle formed was analyzed using computer integrated software. Scanning electron microscopy (SEM) was utilized to display the presence of Aloe vera coating on

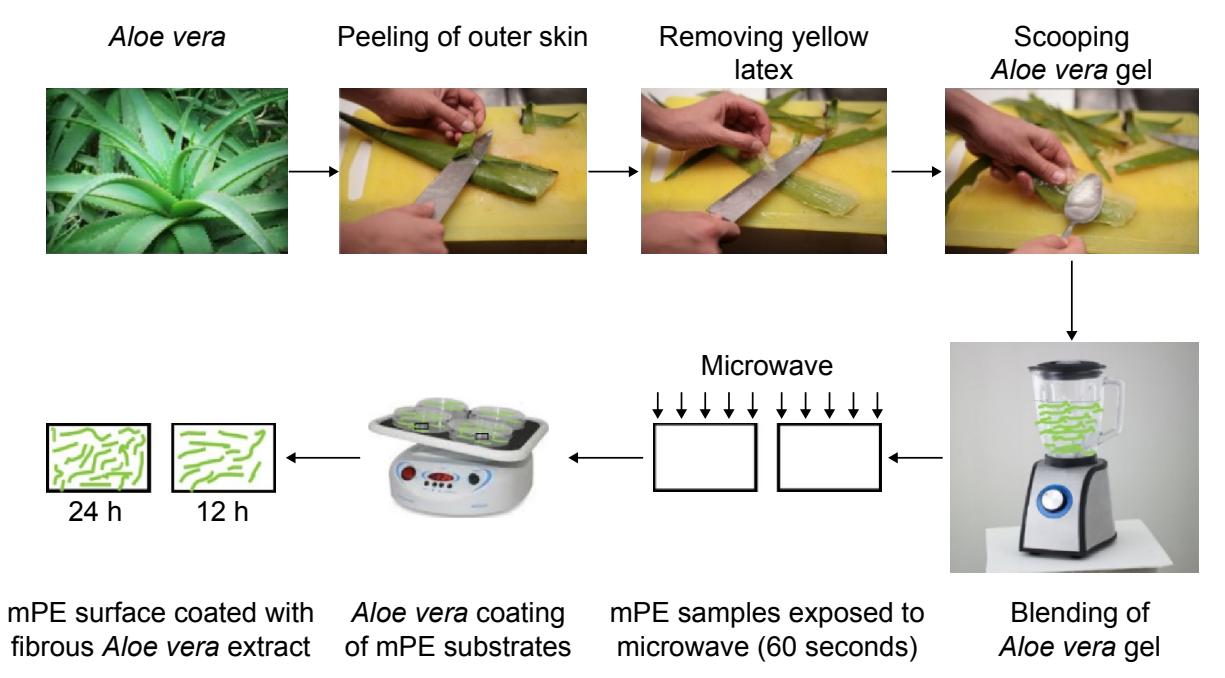

Figure I Steps involved in coating of Aloe vera extract on mPE samples.

Abbreviation: $\mathrm{mPE}$, metallocene polyethylene. 
mPE substrates. For that, samples were sputter coated with gold and imaged through Hitachi Tabletop Microscope (TM3000) coupled with an energy dispersive spectrometry analysis system, at a magnification of $1,500 \times-2,000 \times$. In addition, real-time color images of coated samples and the thickness of Aloe vera coating were determined using Hirox-3D digital microscope (KH-8700). Hirox microscope offers broad range of magnifications from $1 \times$ to $7,000 \times$, and it also produces $3 \mathrm{D}$ images of the scanned area for a better perspective. The images of controls and extract-coated samples were taken at $100 \times, 500 \times$, and $1,000 \times$, respectively, using MGS-2500 REZ lens in standard resolution of 1,200×1,600. Moreover, the distribution of crests and troughs on polymer surfaces was illustrated using built-in 3D profilometry software. Finally, the SPA3800N atomic force microscopy (AFM) was used to perform nanometric surface characterization of $\mathrm{mPE}$ substrates. Scanning was performed in the contact mode over an area of $10 \times 10 \mu \mathrm{m}$ at a rate of $1.50 \mathrm{~Hz}$. Furthermore, SPI3800 software was used to obtain average roughness ( $\mathrm{Ra})$ and root mean square (RMS) values of mPE substrates.

\section{In vitro blood compatibility studies Coagulation assay}

Activated partial thromboplastin time

APTT is a first-line and basic blood compatibility test used to detect the capability of a biomaterial to delay the activation of the coagulation pathway through the intrinsic mechanism, that is, induced by foreign bodies. First, four substrates (two control and two Aloe vera-coated substrates) were incubated at $37^{\circ} \mathrm{C}$ with $100 \mu \mathrm{L}$ of platelet-poor plasma obtained from centrifuged tri-sodium citrate added whole blood. Then, it was activated by adding rabbit brain cephalin $(100 \mu \mathrm{L})$ for 5 minutes. Finally, $0.025 \mathrm{M}$ of calcium chloride was added to the mixture, and the time taken for the appearance of the initial clot was detected by using a steel hook and stopwatch.

\section{Prothrombin time}

On the other hand, PT was calculated to display Aloe veracoated mPE-induced activation of the extrinsic pathway. Like the APTT assay, initially, the samples were preincubated with platelet-poor plasma $(100 \mu \mathrm{L})$ at $37^{\circ} \mathrm{C}$. Then $0.9 \%$ $\mathrm{NaCl}$-thromboplastin (Factor III; $100 \mathrm{~mL}$ )-containing $\mathrm{Ca}^{2+}$ ions was added, and the mixture was slowly stirred using a steel hook until the inception of a clot; the time taken for clot formation was measured using a stopwatch.

\section{Platelet adhesion study}

Adhesion of platelets on polymer surfaces is another threat since this eventually releases clotting factors that end up activating coagulation pathways. This test was performed to scrutinize the ability of Aloe vera-coated surfaces to impede platelet adhesion and activation. Here, PRP was utilized, and the samples were incubated in freshly isolated PRP at $37^{\circ} \mathrm{C}$ for an hour. After that, to fix the adhered platelets on the surface, the samples were incubated with $2 \%$ TritonPSB buffer for 30 minutes at $37^{\circ} \mathrm{C}$, and it was allowed to dry before imaging. Finally, the morphology of adhered platelets and formation of clumps were imaged using a light microscope at $40 \times$.

\section{Hemolysis assay}

To exhibit the percentage of red blood cell (RBC) damage caused by the mPE surface, hemolysis assay (HA) was performed using citrated whole blood. To start with, both control and Aloe vera-coated samples were equilibrated in physiological saline $\left(0.9 \% \mathrm{w} / \mathrm{v}, 37^{\circ} \mathrm{C}, 30\right.$ minutes $)$. Then, it was incubated with a mixture of aliquots of citrated blood ( $3 \mathrm{~mL}$ ) and diluted saline. Subsequently, the samples were incubated in a mixture of blood and distilled water (4:5) to cause complete hemolysis and in physiological saline solution, respectively. The above mixtures were considered as positive (blood: distilled water) and negative (physiological saline) controls, in which samples were incubated for a period of 60 minutes at $37^{\circ} \mathrm{C}$. After retrieving the sample, the mixtures were centrifuged, and the clear supernatant was collected for further analysis. Finally, the absorbance of samples was measured at $542 \mathrm{~nm}$, which directly relates the degree of damage done to RBCs. ${ }^{34}$

\section{Statistical analysis}

All the quantitative experiments were done three times $(n=3)$, and the statistical significance was predicted through one-way ANOVA method. Moreover, the results were expressed as mean \pm standard deviation (SD).

\section{Results and discussion}

The prime duty of a bio-implant is to provide a virtual environment to stimulate and support the regeneration of a desired tissue or an organ system in the human body. To achieve this goal, the artificial material should possess optimum wettability, chemical functionalities, surface energy, roughness, conductivity, and reactivity, which are collectively termed as physicochemical properties. Meanwhile, the range and influence of these factors on promoting rehabilitation have been demonstrated by copious research. So, any type of manufacturing and surface modification technique proposed is essentially directed toward satisfying optimum requirements, thereby achieving the anticipated outcome. Furthermore, 
these surface parameters are also reported to greatly influence crucial aspects of biocompatibility such as blood component interaction and cellular compatibility. Hence, to illustrate the ability of Aloe vera-coated $\mathrm{mPE}$ to produce these essential features, the following studies were performed.

\section{Physicochemical characterization studies} Fourier transform infrared spectroscopy

FTIR is the basic characterization test used to analyze and confirm the presence of appropriate functional groups. The recorded FTIR spectrum is displayed in Figure 2(A), in which the FTIR outline of mPE, used Aloe vera extract, A-12 h-mPE and A-24 h-mPE substrates, are compared. Similar patterns of peaks with different intensities were noted for both control and coated samples at wavelengths $2,916 \mathrm{~cm}^{-1}, 2,848 \mathrm{~cm}^{-1}$, $1,735 \mathrm{~cm}^{-1}, 1,467 \mathrm{~cm}^{-1}, 1,371 \mathrm{~cm}^{-1}, 1,236 \mathrm{~cm}^{-1}, 719 \mathrm{~cm}^{-1}$, and $607 \mathrm{~cm}^{-1}$, respectively. The exhibited twin bands at $2,916 \mathrm{~cm}^{-1}$ and $2,848 \mathrm{~cm}^{-1}$ represent the presence of $\mathrm{C}-\mathrm{H}$ stretch for the alkanes group. The characteristic absorption peak at $1,735 \mathrm{~cm}^{-1}$ was for $\mathrm{C}=\mathrm{O}$ stretch of esters; the peak at $1,467 \mathrm{~cm}^{-1}$ shows the presence of alkane groups $(\mathrm{C}-\mathrm{H}$
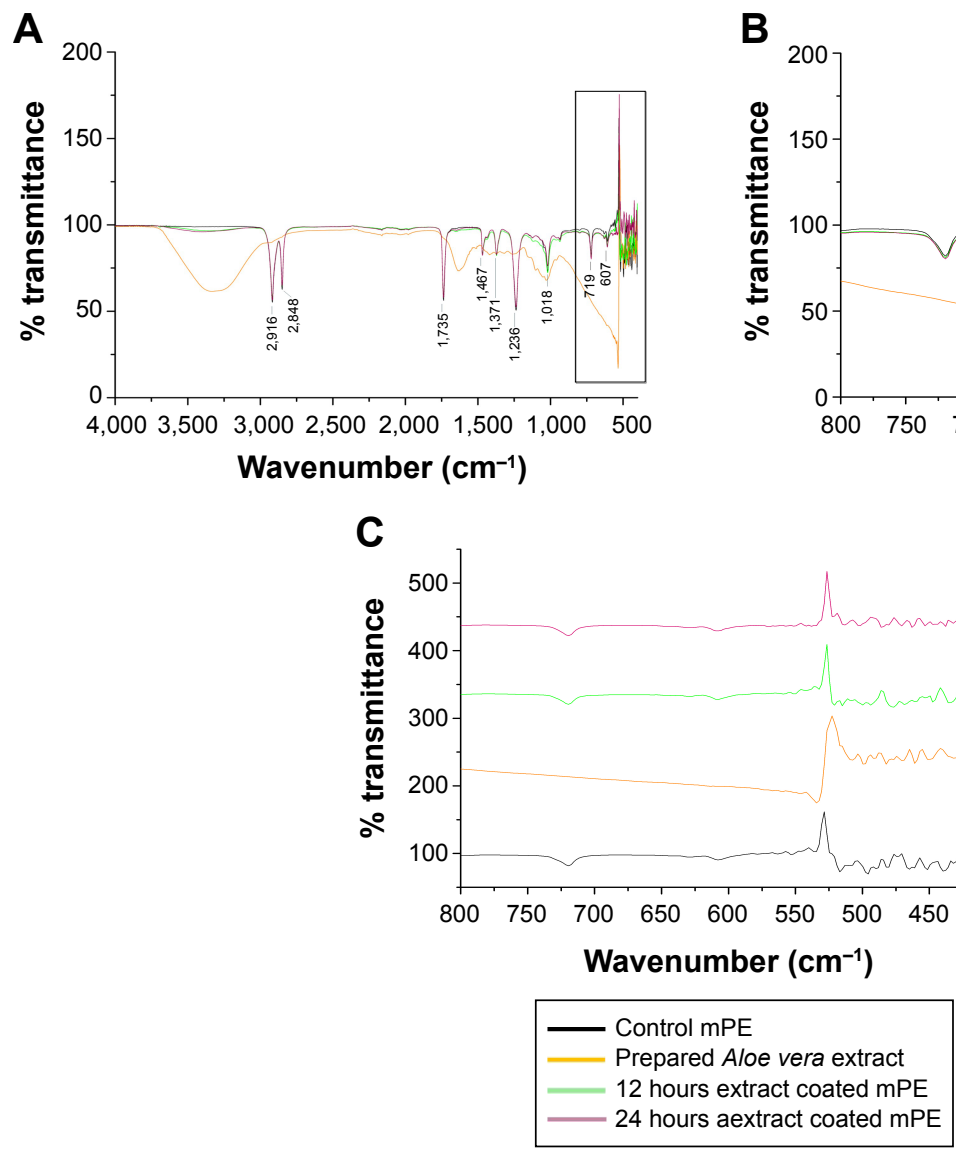

Figure 2 Recorded FTIR spectrum of control and Aloe vera-coated samples.

Notes: (A) Spectrum from 4000-500 $\mathrm{cm}^{-1}$, (B) spectrum from $800-400 \mathrm{~cm}^{-1}$, (C) individual spectrum of substrates at $800-400 \mathrm{~cm}^{-1}$.

Abbreviations: $\mathrm{mPE}$, metallocene polyethylene; FTIR, fourier transform infrared. bending), and the existence of $\mathrm{N}-\mathrm{O}$ stretch is inferred from the peak produced at $1,371 \mathrm{~cm}^{-1}$. Further, the absorption band in the region of $1,236 \mathrm{~cm}^{-1}$ express $\mathrm{C}-\mathrm{O}$ stretch of carboxylic acids (controls), and the crest at $1,018 \mathrm{~cm}^{-1}$ indicates the $\mathrm{C}-\mathrm{O}$ stretch in secondary alcohol. Finally, the peaks at $719 \mathrm{~cm}^{-1}$ and $607 \mathrm{~cm}^{-1}$ displays $\mathrm{C}-\mathrm{H}$ bend of aromatics and alkynes group, respectively.

Though there was no major change in the functional groups, coated mPE samples displayed characteristic absorption spectrum of Aloe vera at $1,735 \mathrm{~cm}^{-1}$ and $1,236 \mathrm{~cm}^{-1}$, indicating the presence of O-acetyl esters; the peaks formed at $1,467 \mathrm{~cm}^{-1}$ and $719 \mathrm{~cm}^{-1}$ revealed the essence of aromatic vibrations of components in Aloe vera gel. ${ }^{8,11}$ Meanwhile, the Aloe vera-coated substrates were also observed to possess shifts in the peaks at far regions of the IR spectra $\left(<600 \mathrm{~cm}^{-1}\right)$, which is displayed clearly in Figure 2B and C. The peaks found at $424 \mathrm{~cm}^{-1}, 439 \mathrm{~cm}^{-1}, 451 \mathrm{~cm}^{-1}, 480 \mathrm{~cm}^{-1}, 516 \mathrm{~cm}^{-1}$ of control were shifted to $422 \mathrm{~cm}^{-1}, 435 \mathrm{~cm}^{-1}, 447 \mathrm{~cm}^{-1}$, $476 \mathrm{~cm}^{-1}, 514 \mathrm{~cm}^{-1}$ for A- $12 \mathrm{~h}-\mathrm{mPE}$ and $426 \mathrm{~cm}^{-1}, 437 \mathrm{~cm}^{-1}$, $453 \mathrm{~cm}^{-1}, 470 \mathrm{~cm}^{-1}, 501 \mathrm{~cm}^{-1}$ for A-24 h-mPE, respectively. These shifts were suspected to appear because of minerals

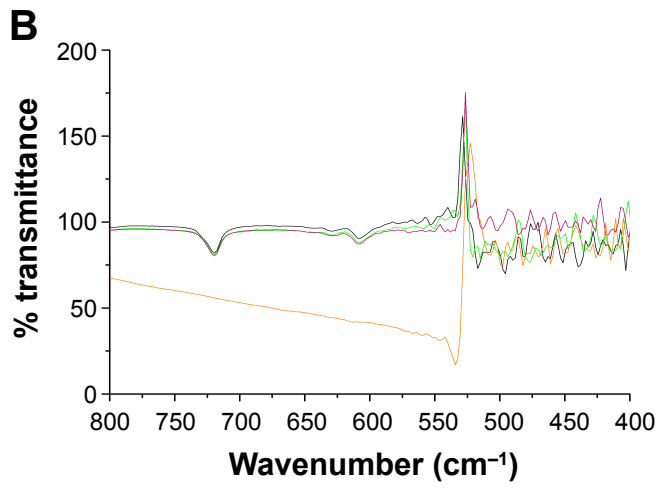


present in Aloe vera gel, which can be clearly concluded from the FTIR fingerprint of extract prepared and the works documented. Additionally, the peaks formed in between $400-500 \mathrm{~cm}^{-1}$ and $500-600 \mathrm{~cm}^{-1}$ demonstrated $\mathrm{Si}-\mathrm{O}$ bending and $\mathrm{C}-\mathrm{H}$ stretch appropriately. ${ }^{35,36}$

Hence, the reported characteristic absorption spectrum and shifts in peaks depict the existence of Aloe vera coating on microwave-treated $\mathrm{mPE}$ samples. The above interpretations also illustrated the presence of active components of Aloe vera gel and chemical functionalities (cell adhesionassisting oxygen groups), which are crucial for mimicking the natural environment and supporting the recovery process.

\section{Contact angle measurement}

Wettability is one of the important surface parameters, which plays a pivotal role in controlling biomolecule adsorption and cellular interaction. In this work, the wettability was measured for both control and coated samples; the experiment was repeated three times, and the contact angle is expressed in mean $\pm \mathrm{SD}$, as displayed in Figure 3 . The mean contact angle calculated for $\mathrm{mPE}$ was $88.43^{\circ}$; the A-12 h-mPE samples had a mean contact angle of $68.13^{\circ}$, whereas the A-24 h-mPE samples showed a drastic increase in wettability by yielding a mean contact angle value of $32.93^{\circ}$ only. So, from the results, we can clearly infer that after extract coating, mPE surfaces become superhydrophilic with contact angle values reduced by more than half compared with control, exclusively in the case of A-24 h-mPE substrates.

This significant improvement is suspected to be due to the water-loving constituents present in Aloe vera gel,

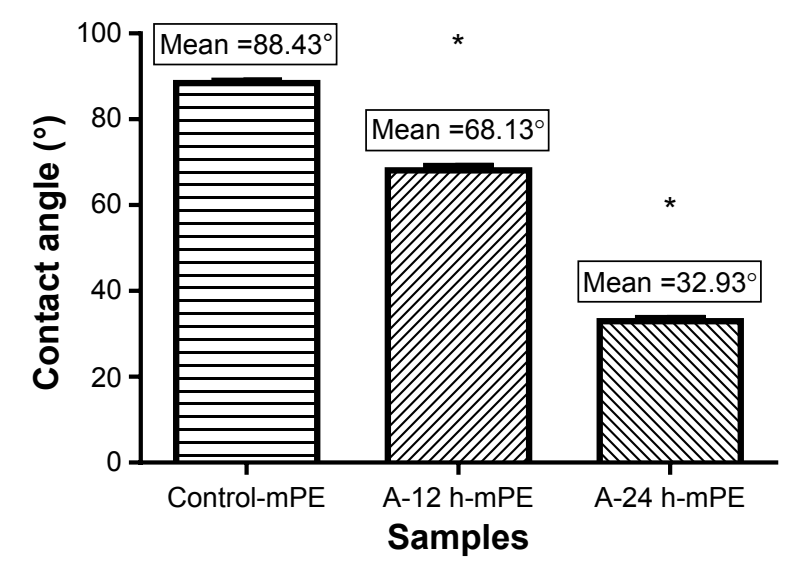

Figure 3 Mean contact angle values of control and coated samples. Note: $*$ Differences between the mean values is significant with respect to control $(P<0.05)$.

Abbreviations: mPE, metallocene polyethylene; A- 12 h-mPE, 12 hours Aloe veracoated; A-24 h-mPE, 24 hours Aloe vera-coated. and it correlates with the results of previous studies. ${ }^{8,11}$ As mentioned earlier, Aloe vera is a natural store house of several polysaccharides, minerals, vitamins, amino acids, and proteins that are basically hydrophilic. The recorded contact angle falls in the optimum range of $70^{\circ}-30^{\circ}$, which has previously been reported in several works ${ }^{37,38}$ of research. A wide variety of cells like fibroblasts, human umbilical vein endothelial cells, osteoblasts, and cardiomyocytes were noted to adhere, proliferate, and differentiate expressively in this range ${ }^{37}$ On the other hand, surfaces with higher wettability also allows the displacement of undesired proteins adhered (like fibrinogen) by cell adhesive serum proteins, such as fibronectin and vitronectin, in contrast to hydrophobic surfaces.$^{38}$ This property of controlling and translating protein adsorption is greatly needed to avoid the complication of blood component activation.

Furthermore, the increased wettability indirectly exhibits the changes in surface energy of mPE samples. In general, surfaces with high energy allow uniform biofilm formation, which in turn produces better spreading of water droplets. ${ }^{39}$ Accordingly, coated samples were observed to facilitate spreading of water droplets, which supports the fact of increased surface energy. The spreading of water droplets on control and Aloe vera-coated samples is illustrated in Figure 4. Therefore, the results of contact angle studies confirm the presence of Aloe vera coating and active biomolecules, as stated in the FTIR test. They also express the possible exploitation of Aloe vera-coated mPE substrates for multipurpose biomedical applications.

\section{SEM analysis}

The SEM micrograph offers a quick view on surface topographical characteristics like roughness, presence of pits, coating, etching, etc. The micrographs were pictured for all samples and the same is compared in Figure 5. The SEM image of untouched mPE recorded at 1,500× showed a clear surface indicating the absence of crests and troughs which ultimately disclosing its poor roughness. In contrast, the 60 -second microwave-treated control imaged at 2,000 $\times$ showed bright white spots depicting the improvement in roughness and formation of pits; these surfaces were reported to improve coating characteristics. Basically, rougher surfaces support better adhesion due to the fact that there is an increase in surface area between coating and substrate. Unlike a flat surface, the area of physical contact is documented to be better in rough surfaces, which ultimately results in mechanical interlocking of coating with substrate, thus enhancing the glazing strength as well. ${ }^{40,41}$ 

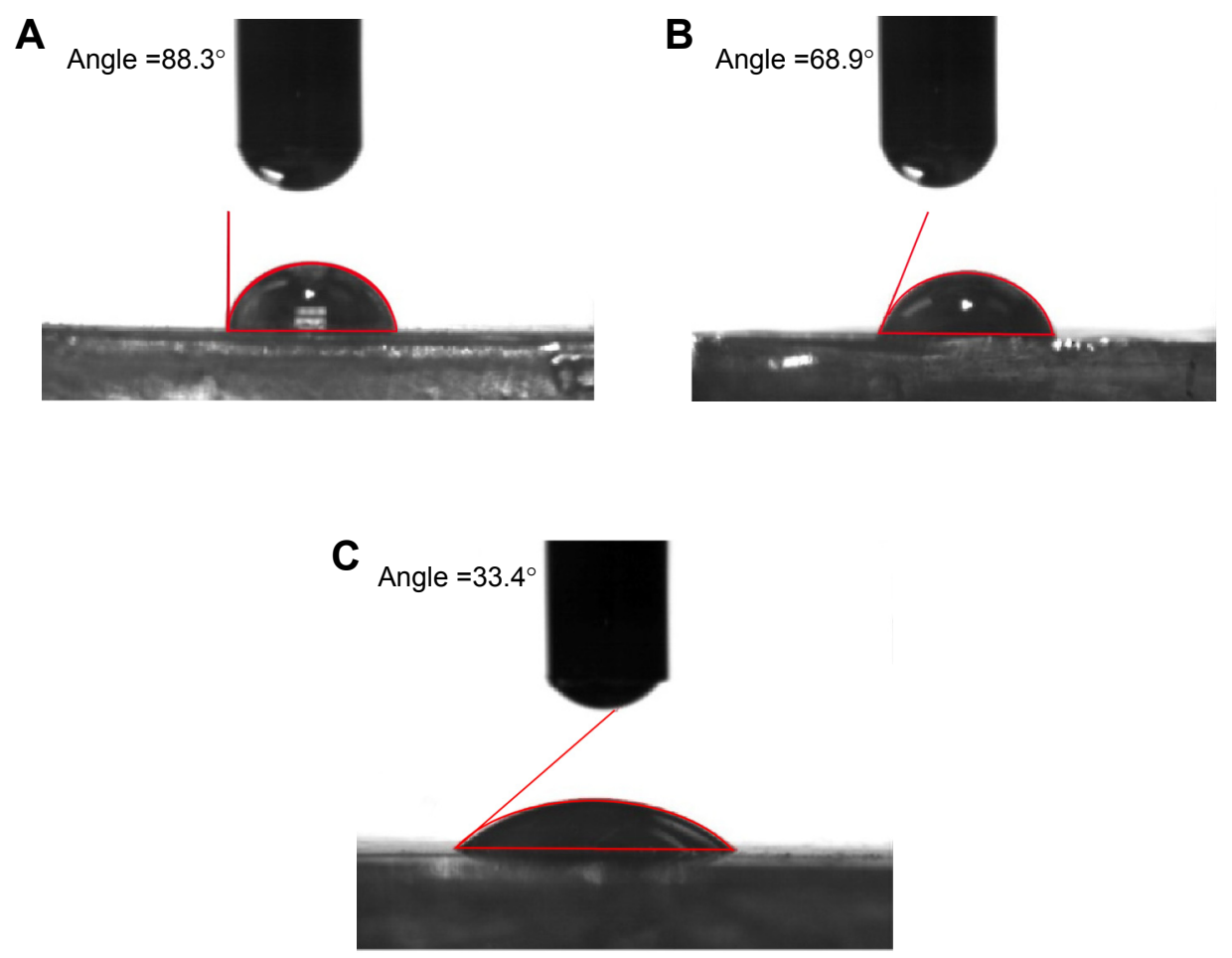

Figure 4 Representative contact angle images showing spreading of water droplet on control and coated mPE samples. Notes: (A) Pristine mPE, (B) 12 hours extract-coated mPE, (C) 24 hours extract-coated mPE. Abbreviation: $\mathrm{mPE}$, metallocene polyethylene.

Therefore, the recorded micrographs evidently support the importance of microwave treatment in augmenting the adhesion and strength of extract coating. Meanwhile, the presence of a fibrous structure was noted from images of
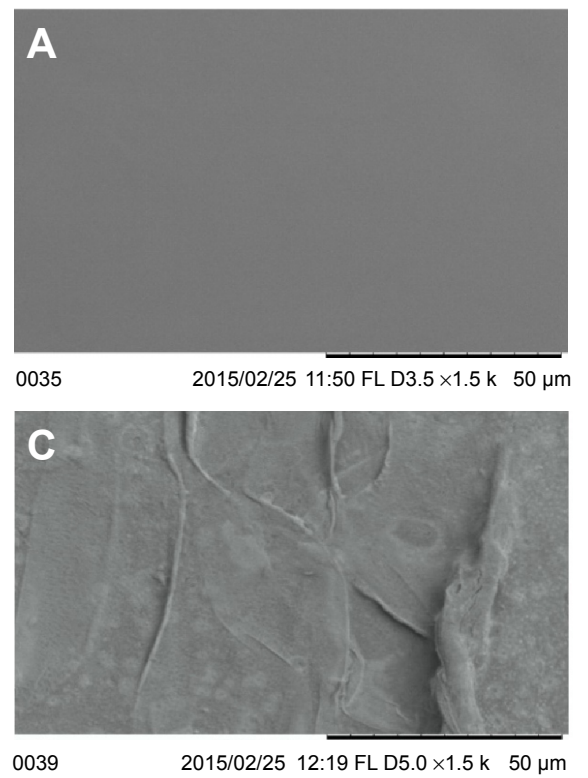

A-12 h-mPE and A-24 h-mPE samples (1,500×), which once again confirms the existence of coating. In addition, the A-24 h-mPE surfaces exhibited dense sedimentation of fibers when compared with A-12 h-mPE, which corroborated
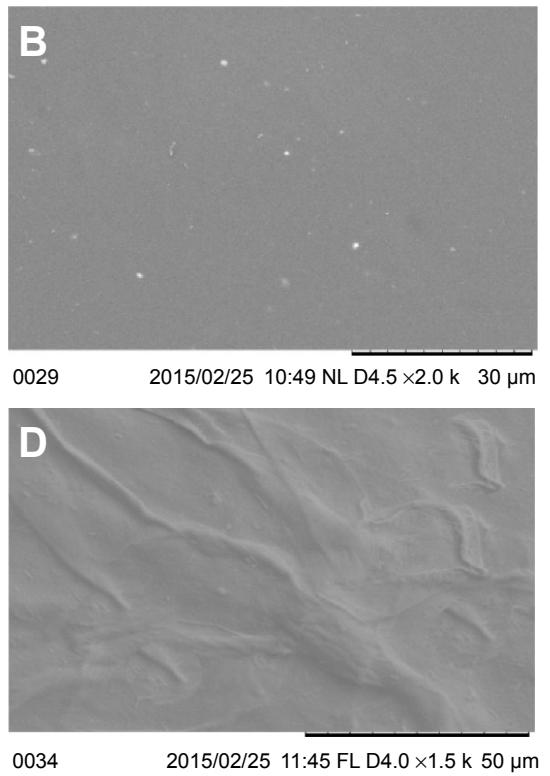

Figure 5 Representative SEM micrographs of control, microwave-treated, and extract-coated mPE samples.

Notes: (A) Pristine mPE at I,500×, (B) 60 seconds microwave-treated mPE at 2,000×, (C) 12 hours Aloe vera-coated mPE at I,500×, and (D) 24 hours Aloe vera-coated $\mathrm{mPE}$ at I,500x.

Abbreviations: $\mathrm{mPE}$, metallocene polyethylene; SEM, scanning electron microscopy. 
the results of contact angle measurement showing higher wettability for samples exposed for a long period. Thus, it can be ascertained that the properties of extract-glazed mPE surfaces enhance with increase in exposure time due to comprehensive settlement of active components present in Aloe vera.

\section{Hirox-3D digital microscope studies}

Inspired by the results of SEM analysis, the micrometric characterization of coated samples and the dispersal of fibrous coating were analyzed with the help of a Hirox $3 \mathrm{D}$ digital microscope. The main advantages of the Hirox microscope are its ability to capture real-time color images and to produce high-resolution two-dimensional (2D) and three-dimensional (3D) views, which meticulously explores the micro-topographical signatures present on the material. Unlike SEM, special preparation of samples, such as coating, is not required in Hirox analysis, which is remarkably suitable for sensitive surfaces. At first, 2D images of pristine-control mPE, A-12 h-mPE, and A-24 h-mPE samples were taken at $100 \times, 500 \times$, and $1,000 \times$, respectively, which are illustrated in Figure 6. From the images, we can clearly observe the presence of homogeneous Aloe vera coating in the form of dense hair-like structures. Like SEM images, the A-24 h-mPE sample exhibited an enriched number of fibers with dense morphology when compared with that of A-12 h-mPE substrates. It again confirmed the time-dependent deposition of active components on microwave-treated mPE. More interestingly, the fibrous coating formed resembles the structure of extra cellular matrix components. Extra cellular matrix is the building block of tissues which contains fibrous biomolecules like collagens, elastin, keratin, fibronectin, laminins, and vitronectin. These structures offer mechanical support to the tissue and regulate several features of cell behavior. These fibers have been reported to control proliferation, migration, and differentiation of cells; further, the orientation of these fibers are dependent on the tissues. Currently, several modern technologies such as electrospinning are mainly aimed at replicating this fibrous environment, thereby enhancing the recovery process by attracting and supporting new cells. ${ }^{42}$ Thus, from the images, we can determine that Aloe veracoated surfaces not only possess essential components for tissue regeneration but also acquire the ability to mimic the biological environment.

Concurrently, the increase in roughness due to microwave treatment and its effect on coating characteristics were also analyzed. The 3D images of substrates recorded at 1,000×, shown in Figure 7, express the presence of crests and troughs. Briefly, number of crests and troughs was increased on coated surfaces when compared with control. To provide a detailed insight, graphical illustration of crests and troughs were obtained for each sample utilizing built-in 3D profilometry software. Using this software, several sketches were randomly drawn on the images recorded at $1,000 \times$, and the one which showed the maximum value was considered for further analysis. After choosing the desired profile, the built-in software generated an excel sheet containing values of coordinates (X, Y, and Z) obtained from 3D geometry, which was exploited to generate a graphical illustration. ${ }^{43}$ Figure 8 compared the fingerprints of untreated and coated samples; the escalation in roughness is inferred due to the microwave treatment and the settlement of fibers on $\mathrm{mPE}$ surface. Apart from the surface roughness, the coating characteristics were also reported to depend on the number of peaks present on the material surface. Excitingly, the microwave-treated samples had more peaks when compared with untouched mPE, which motivated us to check the influence of the microwave-improved properties on the coating thickness achieved.

Hence to determine that, the value of coating thickness present on microwave-treated sample was compared with that of an untreated one. First, the glazed samples were cut into small pieces and vertically placed on the imaging stage using adhesives. When viewed in $100 \times$, the presence of a thin coating was observed, and its thickness was measured by drawing a line. Interestingly, the microwave-treated sample had a coating thickness of $134.8 \mu \mathrm{m}$, while on the untreated $\mathrm{mPE}$, the thickness of Aloe vera extract was only around $99 \mu \mathrm{m}$. So, the Hirox 3D analysis meticulously supports the fact of enhanced coating characteristics of rough surfaces formed by the microwave treatment and also demonstrates the existence of dense fibrous coating similar to SEM images.

\section{AFM analysis}

In addition to coating characteristics, the surface roughness also play a crucial role in determining the behavior of a material within a biological environment alongside surface wettability and energy. The phenomenon that makes the rougher surface highly suitable for cell adhesion and proliferation is the presence of grooves and ridges unlike its counterpart, that is, smooth surfaces. ${ }^{44}$ Further, the range of roughness required for a promising biomaterial was also observed to vary depending on the cells and tissue it intended to assist. For instance, larger cells like osteoblasts were inferred to adhere and proliferate well on surfaces with micrometric roughness. On the other hand, irregularities in the range of the nanoscale were reported to be suitable for 

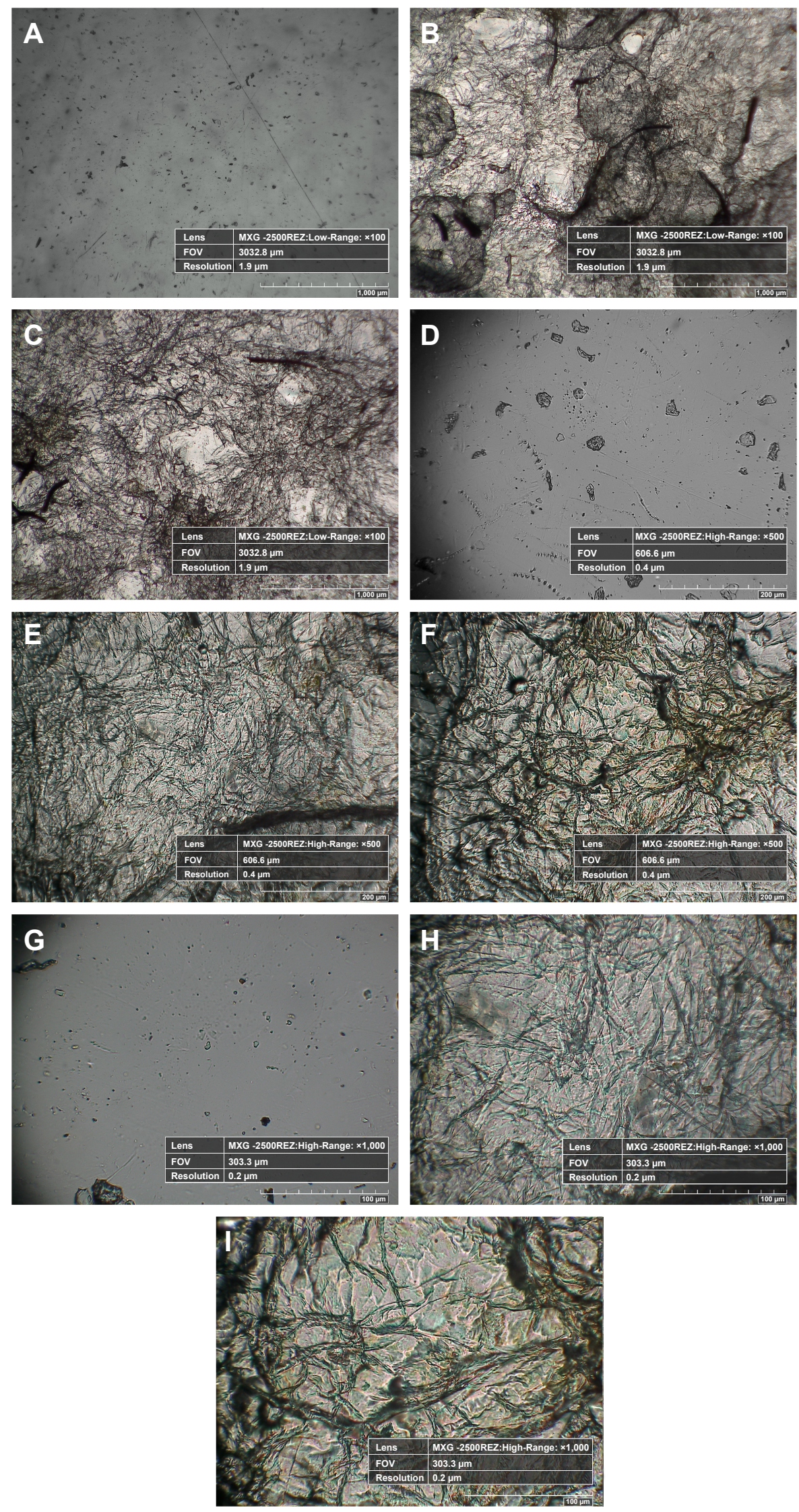

Figure 6 Hirox 2D images of control, 12 hours extract-coated mPE samples and 24 hours extract-coated mPE samples.

Notes: (A), (B), (C) Images taken at 100× (mPE, A-12 h-mPE and A-24 h-mPE, respectively), (D), (E), (F) Images taken at 500×, (G), (H), (I) Images taken at I,000×. Abbreviations: mPE, metallocene polyethylene; D, dimensional; A-12 h-mPE, 12 hours Aloe vera-coated; A-24 h-mPE, 24 hours Aloe vera-coated. 

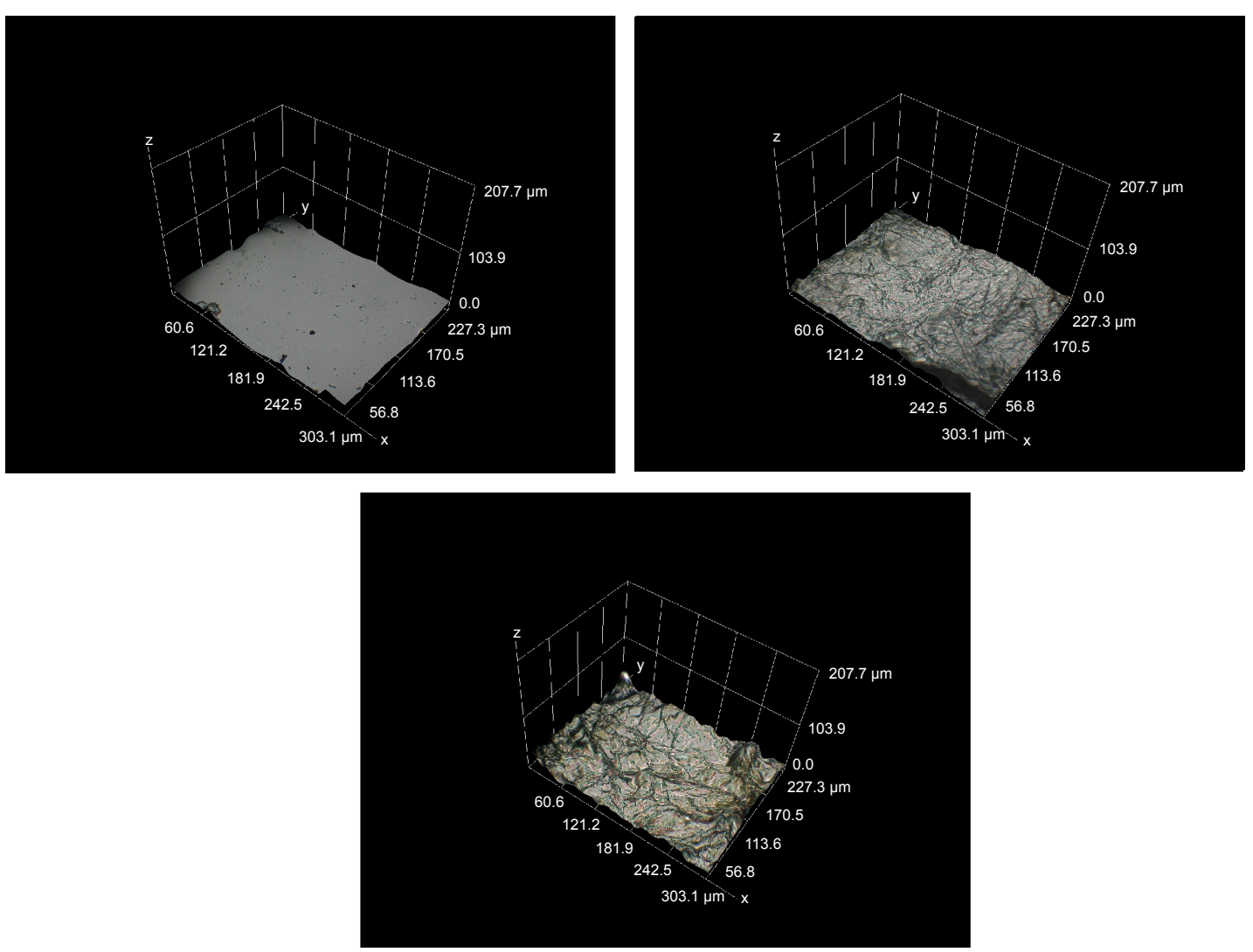

Figure 7 Hirox 3D images of control and extract-coated mPE samples taken at I,000x.

Notes: (A) Pristine mPE, (B) A-I 2 h-mPE, (C) A-24 h-mPE.

Abbreviations: mPE, metallocene polyethylene; D, dimensional; A-12 h-mPE, 12 hours Aloe vera-coated; A-24 h-mPE, 24 hours Aloe vera-coated.

better adhesion, proliferation, and differentiation of fibroblasts, endothelial, and neural cells. ${ }^{37,45}$ Moreover, it was also documented that surface roughness is a vital factor for determining the blood compatible property of a biomaterial, since it has the ability to delay the clot formation. Initially the role of surface roughness in improving coating characteristics was demonstrated using SEM micrographs and Hirox 3D images. Then, AFM is utilized to provide high resolution and 3D nanometric insights of the surface anomalies present on control and Aloe vera-coated samples.

The images were captured on different zones at an area of $10 \times 10 \mu \mathrm{m}$, and the mean value of Ra was statistically calculated using one-way ANOVA. Figure 9 shows the AFM images of pristine and A-24 h-mPE samples. Unlike pristine $\mathrm{mPE}$, the extract-coated substrates emphasized the presence of small pits because of microwave treatment prior to coating, which was also demonstrated in SEM micrographs. In the interim, the mean Ra value for the control was calculated to be $2.069 \mathrm{~nm}$. Conversely, the A-24 h-mPE sample showed a mean Ra of $7.796 \mathrm{~nm}$; this significant improvement confirms the formation of pits and the existence of fibrous coating. Further, the recorded roughness value of coated samples greatly corroborated with the optimum range prerequisite for successful tissue regeneration reported previously. ${ }^{37}$ Thus, from the surface characterization studies, the Aloe vera-coated samples depicted to possess commendable signatures required for a promising biomaterial, which was further established by performing several blood compatibility studies.

\section{In vitro blood compatibility tests}

\section{Coagulation assay}

Hemocompatibility is one of the crucial aspects of medical implants; as soon as a prosthetic material is placed in our body, the first and foremost biological environment it supposed to encounter is what we call the river of life, "blood". It is a complex mixture of RBCs, white blood cells, platelets, plasma proteins, and other essential biomolecules. Whenever a biomaterial comes in contact with the blood stream, it attracts the circulating plasma protein, which results in topping of the material surface with undesired biomolecules. Subsequently, the protein-doped surface activates several clotting factors and platelets moving in the blood stream. Activated platelets then adhere and aggregate on the material surface, which is followed by stimulation of several 

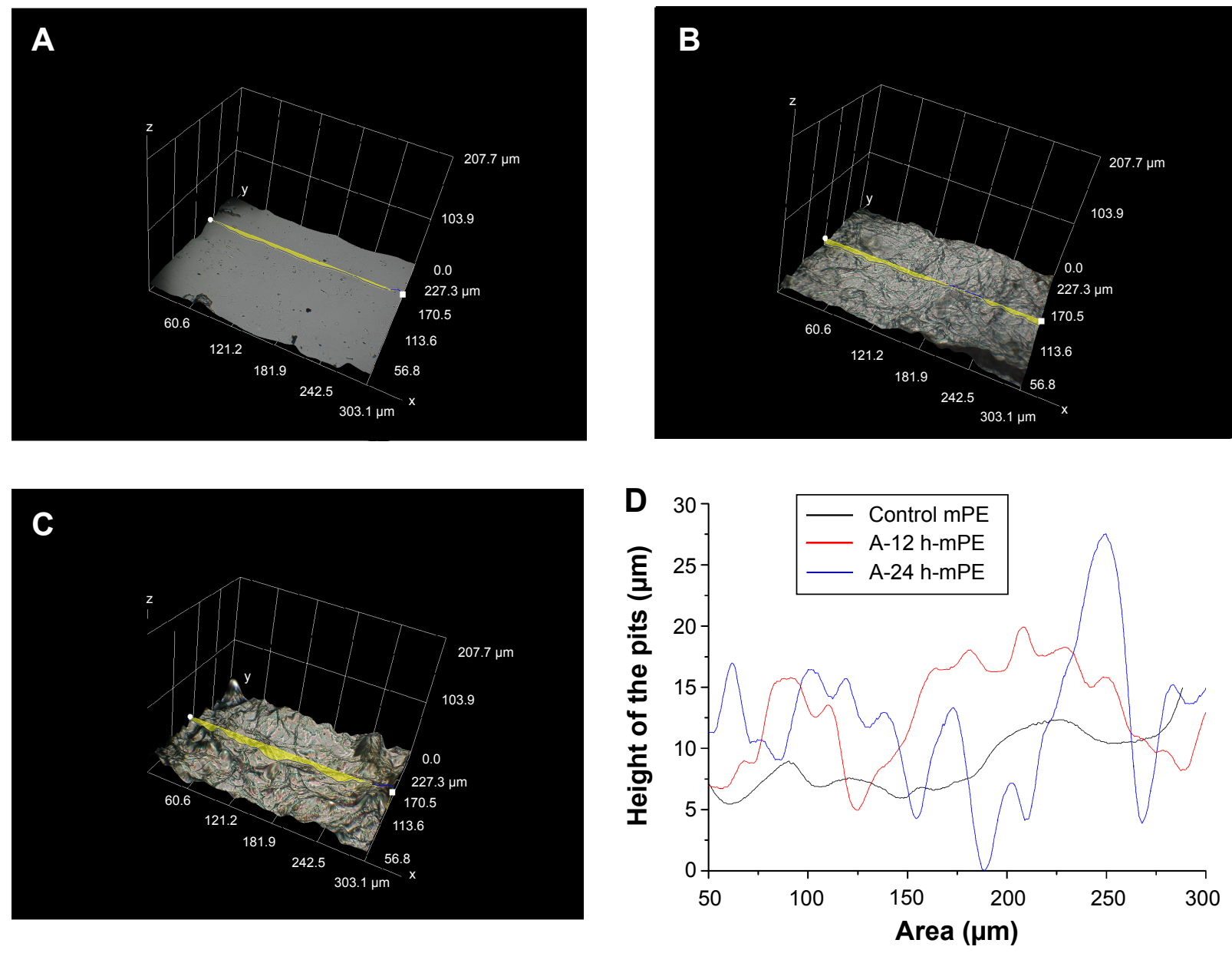

Figure 8 Hirox 3D images of control and extract-coated mPE samples taken at I,000 $\times$ with profiles.

Notes: (A) Pristine mPE, (B) A-12 h-mPE, (C) A-24 h-mPE, (D) outline of crests and troughs in figure (8A, B and C).

Abbreviations: mPE, metallocene polyethylene; D, dimensional; A-12 h-mPE, 12 hours Aloe vera-coated; A-24 h-mPE, 24 hours Aloe vera-coated.

A

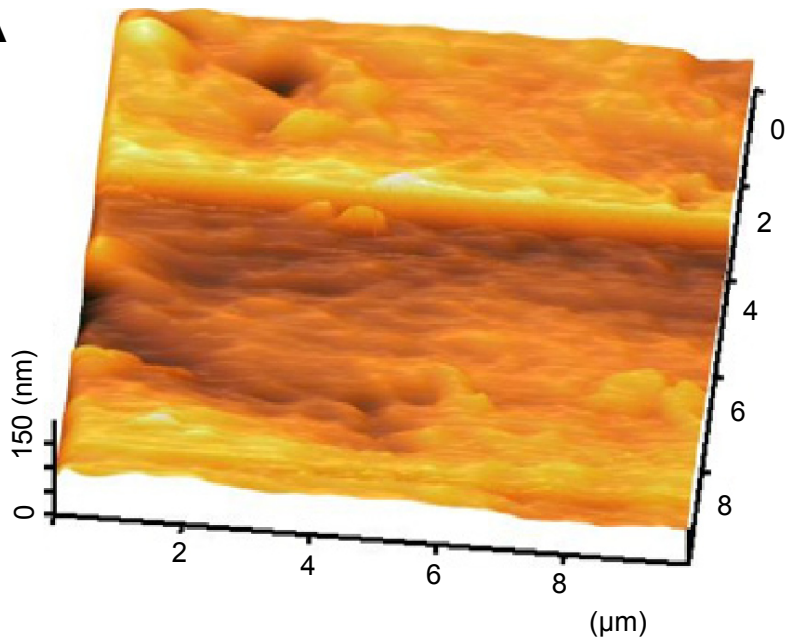

B

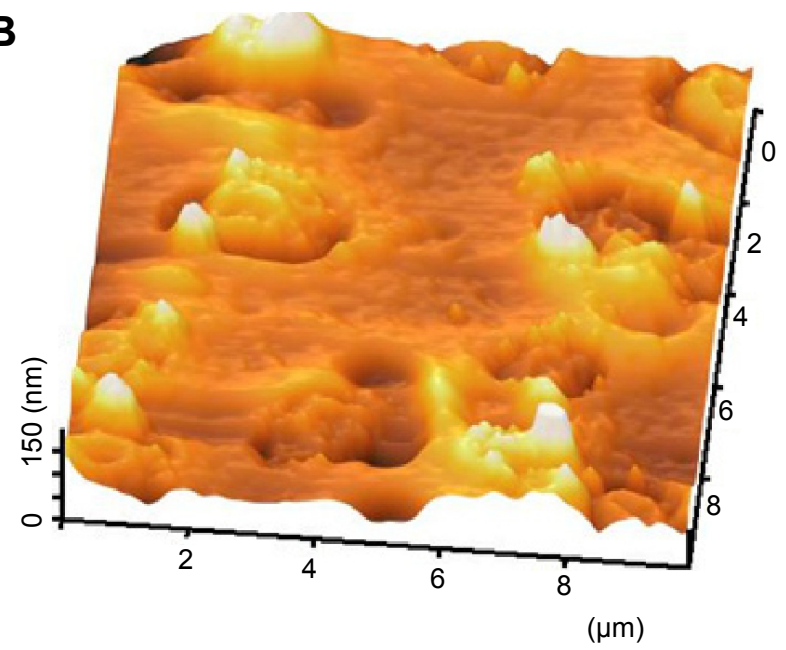

Figure 9 Representative 3D topographical view of control and 24 hours coated sample obtained by AFM.

Notes: (A) Pristine mPE, (B) A-24 h-mPE.

Abbreviations: mPE, metallocene polyethylene; AFM, atomic force microscopy; D, dimensional; A-I2 h-mPE, 12 hours Aloe vera-coated; A-24 h-mPE, 24 hours Aloe veracoated. 
coagulation pathways. The intrinsic and extrinsic pathways are the two main mechanisms greatly engaged in subsequent clot formation. These chain reactions alert our defense system, and paves the way for the occurrence of inflammation and foreign body responses which ultimately ends in rejection of the implant. ${ }^{16,17}$

However, a biomaterial with commendable surface features has been reported to avert these complications either by halting protein adsorption or by allowing the displacement of undesired proteins adhered with cellular compatible biomolecules, as mentioned previously. ${ }^{37,38}$ This property not only saves the implant from rejection but also boosts its job of assisting diseased tissue. The characterization studies carried out so far reveal the improvement of $\mathrm{mPE}$ surface properties after extract coating. Aloe vera, used in this work, consists of various natural anti-inflammatory agents in the form of sugars, amino acids, lipids, active enzymes, and salicylic acid. And the extract has been exploited as a powerful wound healing agent for several centuries. Thus, in order to disclose the ability of Aloe vera-improved mPE surfaces in combating the expected blood mediated complications, in vitro coagulation assays were performed.

\section{Activated partial thromboplastin time}

APTT assay was performed on both control and coated $\mathrm{mPE}$ samples. The experiment was repeated three times $(n=3)$ to check the reproducibility of outcomes observed. Statistical analysis was performed through one-way ANOVA, and the mean values are illustrated in Figure 10. The control $\mathrm{mPE}$ had

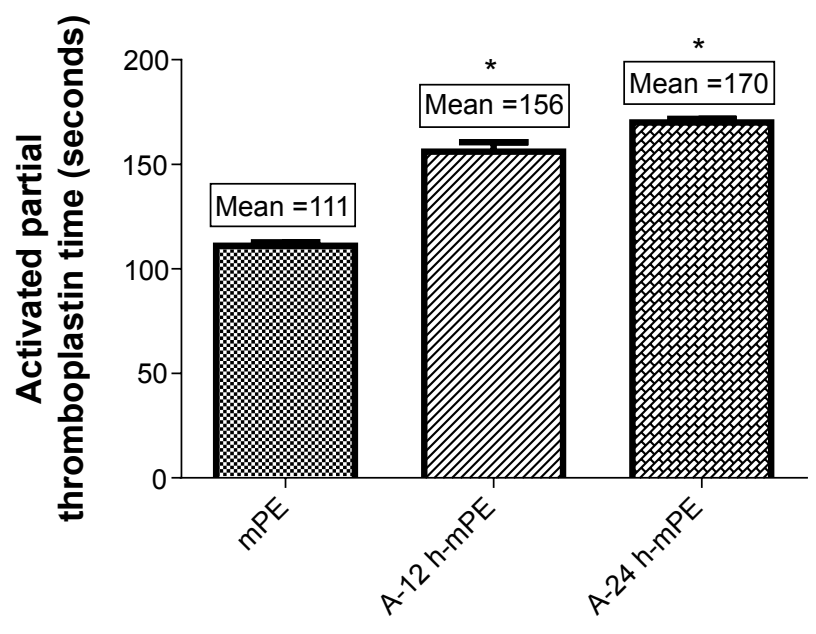

Duration of treatment (minutes)

Figure 10 Comparison of activated partial thromboplastin time of control and coated $\mathrm{mPE}(\mathrm{n}=3)$.

Notes: Values shown are mean \pm SD and the difference in mean is significant with $P<0.05$. *Differences between the mean values is significant with respect to control $(P<0.05)$.

Abbreviations: mPE, metallocene polyethylene; A- 12 h-mPE, 12 hours Aloe veracoated; A-24 h-mPE, 24 hours Aloe vera-coated. a mean clotting time of 111 seconds, whereas the Aloe veracoated sample demonstrated a notable delay in clot formation. The A-12 h-mPE surfaces were able to avert clot formation for a period of 156 seconds, and the A-24 h-mPE samples exhibited an increased clot formation time of 170 seconds. The statistical analysis showed a significant difference $(P<0.05)$ between control and coated samples.

\section{Prothrombin time}

Prothrombin time was calculated to illustrate the ability of Aloe vera-coated mPE surfaces to delay the formation of clots through the extrinsic pathway. Like APTT, the experiment was repeated three times, and the mean value obtained for each sample was considered as its PT. As expected, the coated samples showed a better delay in clot formation when compared with controls. The A-12 h-mPE samples had a mean clotting time of 36.1 seconds compared with 39 seconds for A-24 h-mPE substrates, while for controls, the mean PT was 27.23 seconds. Moreover, the statistical analysis carried out using one-way ANOVA showed a significant difference $(P<0.05)$ between control and extract-coated samples, which is represented in Figure 11. The above outcomes illustrate the ability of Aloe vera coating to delay the development of clots through contact activation, that is, both intrinsic and extrinsic pathways.

\section{Platelet adhesion studies}

Adhesion of platelets is another important phenomenon, which is actively involved in stimulation of undesired

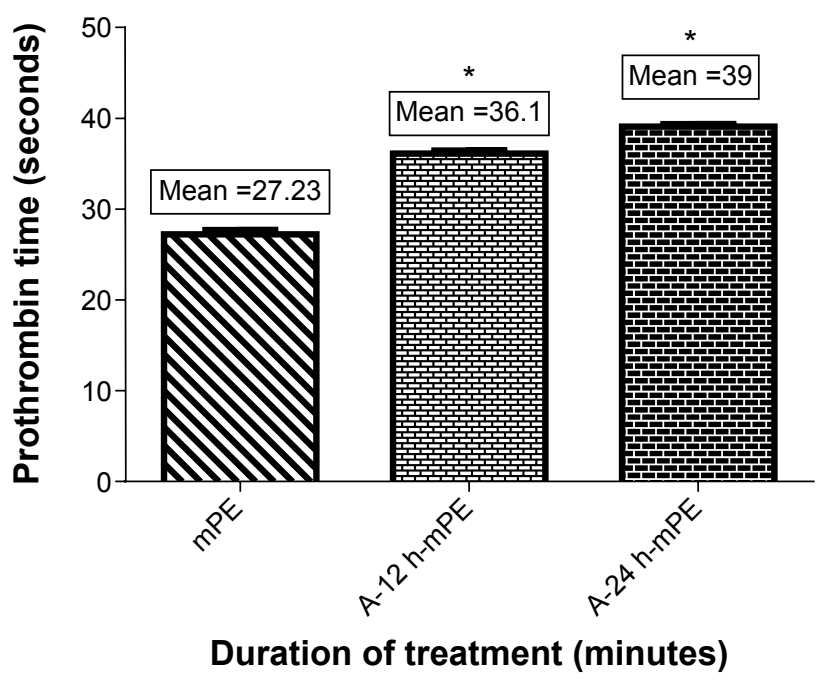

Figure II Comparison of prothrombin time of pristine and extract-coated metallocene polyethylene $(n=3)$.

Note: Values shown are mean \pm SD and the difference in mean is significant with $P<0.05$. *Differences between the mean values is significant with respect to control $(P<0.05)$.

Abbreviations: mPE, metallocene polyethylene; A- 12 h-mPE, 12 hours Aloe veracoated; A-24 h-mPE, 24 hours Aloe vera-coated. 
hemo-complications. In this work, the platelet-repellent property of Aloe vera-coated substrates were explored using PRP. Images of all samples taken using a light microscope at $40 \times$ were compared in Figure 12. The platelets were observed to readily adhere and aggregate extensively on pristine $\mathrm{mPE}$, whereas on A-12 h-mPE samples, the adhesion was very minimal, and the aggregation also reduced significantly. On the other hand, the A-24 h-mPE samples exhibited an excellent ability to combat platelet attachment by reducing the adhesion percentage less than one (approximately). This significant improvement may indicate the presence of antioxidants and platelet-repellent biomolecules available in Aloe vera on coated samples. The same was reported in the in vivo studies performed by Singh and Fahim. ${ }^{46}$ In the case of pristine samples, the absence of coating and lack of required topographical characteristics pave the way for deposition of unspecific plasma proteins. It in turn facilitates the activation, adhesion, and aggregation of platelets. In addition to Aloe vera extract, the presence of nano-roughness is also suspected to influence the platelet-repellent property of the modified
mPE surface, which is highly desirable in temporary and permanent blood-contacting medical devices.

\section{Hemolysis assay}

Apart from deterring clot formation, a material should also demonstrate good compatibility with blood cells. RBC is the abundant cell type present in the blood, and a commendable relationship with these cells will serve as a marker for better cellular compatibility of an appropriate biomaterial. For determining this, HA was performed, and the absorbance value recorded at $542 \mathrm{~nm}$ related the damage caused to RBCs upon biomaterial interaction. The mean absorbance elicited by the control was 0.018 . Interestingly, for A-12 h-mPE, the mean absorbance was 0.015 , and for A-24 h-mPE samples, it was 0.0065 (Figure 13), which is only a quarter percentage of uncoated samples. This reduction in absorbance with increasing time of Aloe vera exposure reveals the enhancement in compatible properties of $\mathrm{mPE}$ surfaces after coating. Hence, from the above interpretation, we can conclude that the Aloe vera-glazed mPE substrates
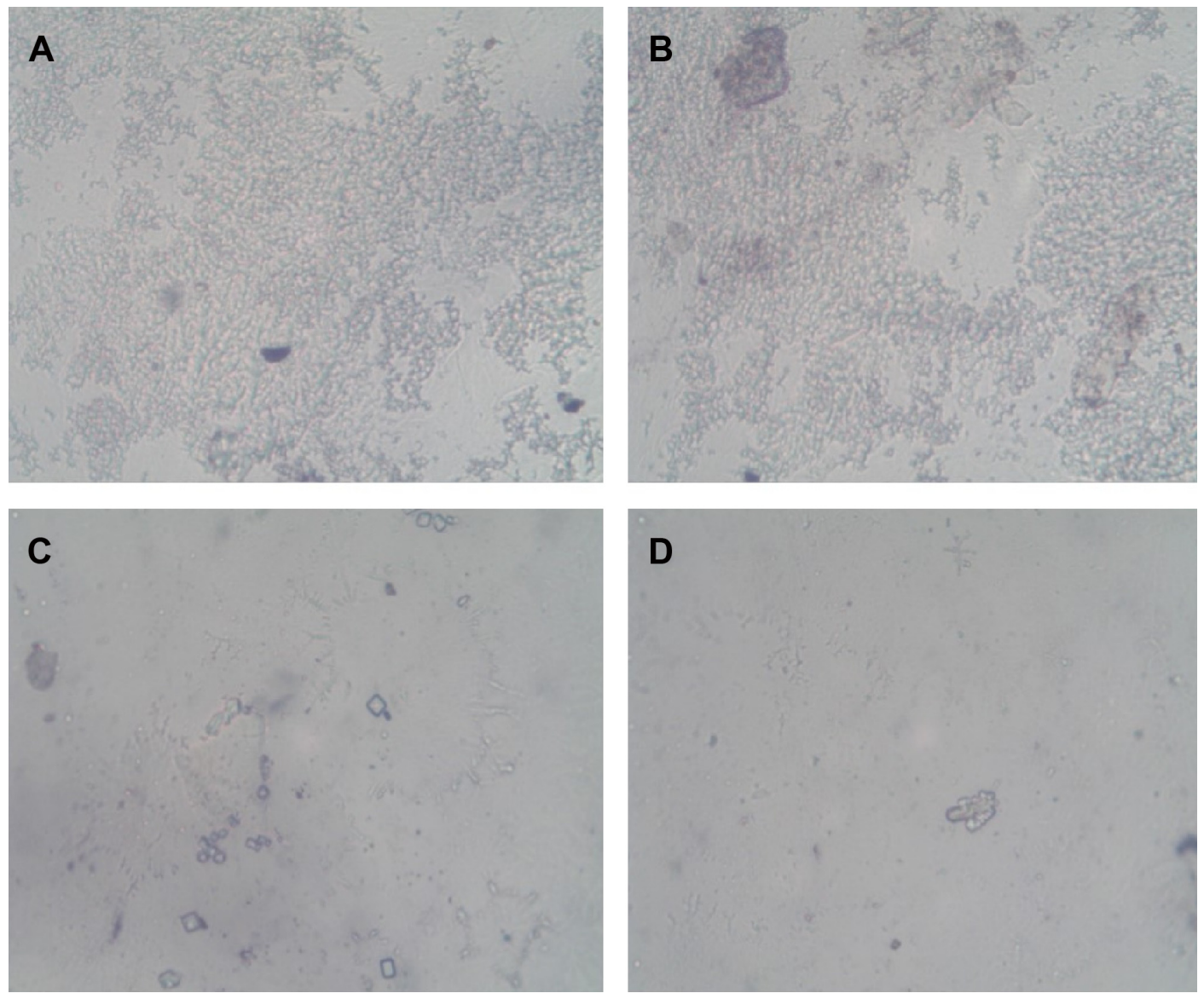

Figure 12 Light microscopic images showing adhered platelets on control and extract-coated $\mathrm{mPE}$.

Notes: (A), (B) Pristine mPE, (C) A-12 h-mPE, (D) A-24 h-mPE.

Abbreviations: mPE, metallocene polyethylene; A- 12 h-mPE, 12 hours Aloe vera-coated; A-24 h-mPE, 24 hours Aloe vera-coated. 


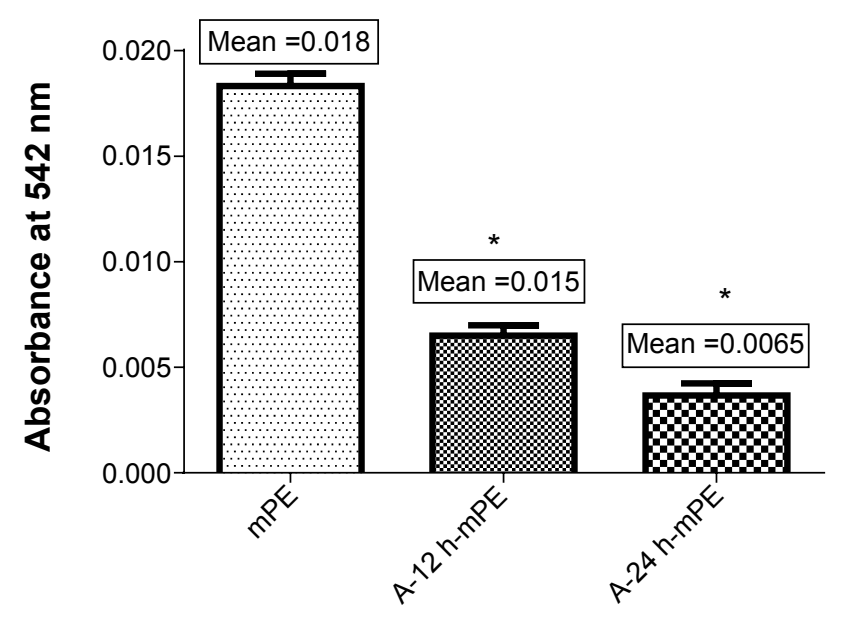

\section{Duration of treatment (minutes)}

Figure 13 Comparison of absorbance of $\mathrm{mPE}$ and Aloe vera-coated metallocene polye-thylene $(n=3)$.

Notes: Values are shown in mean $\pm S D$, and differences in the mean are significant $(P<0.05)$. *Differences between the mean values is significant with respect to control $(P<0.05)$.

Abbreviations: mPE, metallocene polyethylene; A- 12 h-mPE, 12 hours Aloe veracoated; A-24 h-mPE, 24 hours Aloe vera-coated.

acquire the ability to avert expected complications in the biological environment.

\section{Conclusion}

Research on exploring novel materials and surface modification techniques in the field of biomaterials has been going on for decades. In this study, the biocompatibility of mPE, a polymer in clinical utilization, is improved by coating with Aloe vera extract with the help of microwaves. The results of surface characterization studies such as FTIR, contact angle, SEM, Hirox 3D, and AFM analysis demonstrate the achievement of optimum wettability, surface energy, and roughness followed by the coating of dense fibrous Aloe vera extract. Further, the importance of the microwave treatment in influencing the surface morphology and improving the coating thickness is also demonstrated using SEM micrographs and Hirox 3D images. Ultimately, the active biomolecules of Aloe vera present on the coated surface has enriched the blood compatibility of $\mathrm{mPE}$ as demonstrated through APTT, PT, platelet adhesion, and HA studies. Hence, from the results obtained in this work, it is concluded that Aloe vera-glazed mPE surfaces have great potential to support and guide the tissue rehabilitation process. Further, this cost-effective system can be plausibly employed for multifaceted tissue engineering and biomedical applications, especially in the field of maxillofacial, orthopedic, and cardiovascular implants.

\section{Acknowledgment}

This work was supported partly by the Ministry of Higher Education Malaysia, with the Grant Vot No R.J130000.7809.4F444 and the Ref No: PY/2014/03167.

\section{Disclosure}

The authors declare that they have no conflict of interest in this work.

\section{References}

1. Josias HH. Composition and applications of Aloe vera leaf gel. Molecules. 2008;13:1599-1616.

2. Amar S, Resham V, Saple DG. Aloe vera; a short review. Indian $J$ Dermatol. 2008;53(4):163-166.

3. Herzberg F. Aloe Vera: An International Success Story, Nutraceuticals world, 2003. Available from: http;/www.nutraceuticalsworld.com/ issues/2003-05/view_features/aloe-vera-an-international-successstory/

4. Davis RH, Di Donato JJ, Hartman GM, Hass RC. Anti-inflammatory and wound healing activity of a growth substance in Aloe vera. J Am Podiatr Med Assoc. 1994;84(2):77-81.

5. Heggers JP. Beneficial effect of aloe on wound healing in an excisional wound healing model. J Altern Complement Med. 1996;2(2):271-277.

6. Davis RH, Leitner MG, Russo JM, Byrne ME. Wound healing. Oral and topical activity of Aloe vera. JAMA. 1989;79(11):559-562.

7. Yagi A, Kabash A, Mizuno K, Moustafa SM, Khalifa TI, Tsuji H. Radical scavenging glycoprotein inhibiting cyclooxygenase-2 and thromboxane A2 Syn- thase from Aloe vera gel. Planta Med. 2003;69(3):269-271.

8. Agnes Mary S, Giri Dev VR. Electrospun herbal nanofibrous wound dressings for skin tissue engineering. J Text Ind. 2014.

9. Zhang Y, Yang D, Kong Y, Wang X, Pandoli O, Ga G. Synergetic antibacterial effects of silver nanoparticles@Aloe vera prepared via a green method. Nano Biomed Eng. 2010;2(4):252-257.

10. Shreya M, Amita H, Uttiya D, Paulomi B, Naba Kumar M. Biosynthesis of silver nanoparticles from Aloe vera leaf extract and antifungal activity against Rhizopus sp. and Aspergillus sp. Appl Nanosci. 2015;106(8): 886-895.

11. Suganya S, Venugopal J, Ramakrishna S, Lakshmi BS, Dev VR. Naturally derived biofunctional nanofibrous scaffold for skin tissue regeneration. Int J Biol Macromol. 2014;68:135-143.

12. Karuppuswamy P, Venugopal J, Balchandar N, et al. Functionalized hybrid nanofibers to mimic native ECM for tissue engineering applications. Appl Surf Sci. 2014;322:162-168.

13. Sridhar R, Ravanan S, Venugopal JR, et al. Curcumin- and natural extract-loaded nanofibres for potential treatment of lung and breast cancer; in vitro efficacy evaluation. J Biomater Sci Polymer Ed. 2014; 25(10):985-998.

14. MarketsandMarkets. Global Bio-Implants Market worth $\$ 134.3$ Billion by 2017. Available from: http://www.marketsandmarkets.com/ PressReleases/bio-implants.asp. Accessed January 25, 2015.

15. Online report: Transparency Market reports. Bio-Implants Market - Global Industry Analysis, Size, Share And Forecast 20122018. Available from: http://www.transparencymarketresearch.com/ bio-implants-market.html. Accessed February 18, 2015.

16. Stevens KNJ. Blood-Contacting Biomaterials for Critical Clinical Applications. The Netherlands: Maastricht University: 2011.

17. Liu X, Yuan L, Tang Z, et al. Blood compatible materials state of the art. J Mater Chem. B. 2014;2:5718.

18. Hoffman AS. Surface modification of polymers. Chin J Polymer Sci. 1995;13:1-9.

19. Jaganathan SK, Arunpandian B, Muthu Vignesh V, et al. Review Radiation-induced surface modification of polymers for biomaterial application. J Mater Sci. 2014. 
20. Lipsitt B. Performance Properties of Metallocene Polyethylene, EVA and Flexible PVC Films. Medical Plastics and Biomaterials Magazine; 1998.

21. Lipsitt B. Metallocene polyethylene films as alternatives to flexible PVC film for medical device fabrication. In: Proceedings of the Society of Plastics Engineers 55th Annual Technical Conference (ANTEC '97), SPE, Brookfield, Conn, USA; 1997:2854-2858.

22. Kutz M. Handbook of Materials Selection. Wiley; 2002:1520. [ISBN: 978-0-471-35924-1].

23. Mohandas H, Sivakumar G, Palaniappan K, Jaganathan SK, Supriyanto E. Microwave-assisted surface modification of metallocene polyethylene for improving blood compatibility. Biomed Res Int. 2013:1-7.

24. Jaganathan SK, Mohandas H, Sivakumar G, et al. Enhanced blood compatibility of metallocene polyethylene subjected to hydrochloric acid treatment for cardiovascular implants. Biomed Res Int. 2014:1-7.

25. Buchkoa CJ, Kozloffb KM, Martinc DC. Surface characterization of porous, biocompatible protein polymer thin films. Biomaterials. 2001;22:1289-1300.

26. Chena H, Yuana L, Songa W, Wub Z, Lia D. Biocompatible polymer materials, role of protein-surface interactions. Prog Polym Sci. 2008;33:1059-1087.

27. Wang X, Cooper S. Adhesion of endothelial cells and endothelial progenitor cells on peptide-linked polymers in shear flow. Tissue Eng A. 2013;19:1113-1121.

28. Hall B, Richard le E, Masayoshi K, Dennis C. Biomembranes as models for polymer surfaces V Thrombelastographic studies of polymeric lipids and polyesters. Biomaterials. 1989;10:219-224.

29. Asadinezhad A. Polysaccharides coatings on medical-grade PVC, a probe into surface characteristics and the extent of bacterial adhesion. Molecules. 2010;15:1007.

30. Zhou H, Nabiyouni M, Bhaduri SB. Microwave assisted apatite coating deposition on Ti6Al4V implants. Mater Sci Eng C. 2013;33:4435-4443.

31. Irzh A, Gedanken A. A microwave assisted process for coating polymer and glass surfaces with semiconducting $\mathrm{ZnO}$ submicron particles. $J \mathrm{Appl}$ Polymer Sci. 2009;113(3):1773-1780.

32. Irzh A, Perkas N, Gedanken A. Microwave-assisted coating of PMMA beads by silver nanoparticles. Langmuir. 2007;23:9891-9897.

33. Tuval T, Gedanken A. A microwave-assisted polyol method for the deposition of silver nanoparticles on silica spheres. Nanotechnology. 2007;18:255-601.
34. Amarnath LP, Srinivas A, Ramamurthi A. In vitro hemocompatibility testing of UV-modified hyaluronan hydrogels. Biomaterials. 2006;27:1416-1424.

35. Povnn AS. The use of infrared spectra for the determination of minerals. Am Mineral. 1978;63:956-959.

36. Christian Menno M, Pejcic B, Esteban L, Delle Piane C, Raven M. Infrared attenuated total reflectance spectroscopy: an innovative strategy for analyzing mineral components in energy relevant systems. Sci Rep. 2014;4:6764.

37. Chang HI, Wang Y. Regenerative Medicine and Tissue Engineering-Cells and Biomaterials Cell Responses to Surface and Architecture of Tissue Engineering Scaffolds. Croatia: Intech publications; 2011:1-21.

38. Arima Y, Iwata $\mathrm{H}$. Effect of wettability and surface functional groups on protein adsorption and cell adhesion using well-defined mixed self-assembled monolayers. Biomaterials. 2007;28:3074-3082.

39. Poncin-Epaillard F, Legeay G. Surface engineering of biomaterials with plasma techniques. J Biomater Sci Polymer Ed. 2003;14:1005-1028.

40. Roper HJ. The Effect of Peak Count of Surface Roughness on Coating Performance. Available from: http://www.paintsquare.com/library/ articles/the_effect_of_peak_count_of_surface_roughness_on_coating_performance.pdf. Accessed February 28, 2015.

41. Justina V, Kristina U, Gintaras K. The effects of surface roughness on adhesion strength of coated ash (Fraxinus excelsior L.) and Birch (Betula L.) Wood. Mater Sci. 2012;4:18.

42. Beachleya V, Wen X. Polymer nanofibrous structures: fabrication, biofunctionalization, and cell interactions. Prog Polym Sci. 2010;35(7):868-892.

43. Pacifici E, Bossu M, Giovannetti A, La Torre G, Guerra F, Polimeni A. Surface roughness of glass ionomer cements indicated for uncooperative patients according to surface protection treatment. Ann Stomatol. 2013 4(3-4):250-258.

44. Deligianni DD, Katsala ND, Koutsoukos PG, Missirlis YF. Effect of surface roughness of hydroxyapatite on human bone marrow cell adhesion, proliferation, differentiation and detachment strength. Biomaterials. 2001;22:87-96.

45. Li L, Crosby K, Sawicki M, Shaw LL, Wang Y. Effects of surface roughness of hydroxyapatite on cell attachment and proliferation. J Biotechnol Biomater. 2012;2:150.

46. Singh $\mathrm{S}$, Fahim MA. In vivo antiplatelet aggregatory activity of Aloe vera juice on mice cerebral micro vessels. Iran J Pharm Res. 2004;3:70.
International Journal of Nanomedicine

\section{Publish your work in this journal}

The International Journal of Nanomedicine is an international, peerreviewed journal focusing on the application of nanotechnology in diagnostics, therapeutics, and drug delivery systems throughout the biomedical field. This journal is indexed on PubMed Central, MedLine, CAS, SciSearch $®$, Current Contents $\AA /$ Clinical Medicine,

\section{Dovepress}

Journal Citation Reports/Science Edition, EMBase, Scopus and the Elsevier Bibliographic databases. The manuscript management system is completely online and includes a very quick and fair peer-review system, which is all easy to use. Visit http://www.dovepress.com/ testimonials.php to read real quotes from published authors. 